\title{
Análisis de la innovación financiera como un proceso generador de ingresos en las cooperativas de ahorro y crédito del segmento 1 de la provincia de Chimborazo.
}

Title Analysis of financial innovation as an income generating process in the saving and credit cooperatives of segment 1 of Chimborazo province.

Iván Patricio Arias González. ${ }^{1}$, Mariana Isabel Puente Riofrío. ${ }^{2}$ \& Mónica del Pilar Vallejo Villalva. ${ }^{3}$

\begin{abstract}
.
DOI: https://doi.org/10.33262/concienciadigital.v4i1.2.1588

Introduction: Financial innovation, being considered a tool that allows offering faster and lower-cost financial products and services, is directly linked to the generation of income for entities in the financial sector. Objective: Analyze the incidence of financial innovation in the generation of income in the savings and credit cooperatives segment 1 of the Chimborazo province, considering variables such as ATMs, deposits, placements, number of financial operations and the income generated in the period 2018 - 2020. Methodology: The research approach was quantitative, the inductive deductive method allowed to analyze the data obtained from the variables, a linear regression model was applied through the STATA 16.1 software. Results: The financial innovation known as ATMs shows a $36 \%$ increase in the income of the savings and credit cooperatives in segment 1. Conclusion: According to the results obtained, it is concluded that there is a

\footnotetext{
1 Universidad Nacional de Chimborazo, Carrera de Contabilidad y Auditoría, Chimborazo. ivan.arias@unach.edu.ec. Orcid ID: 0000-0002-6842-848X

2 Universidad Nacional de Chimborazo, Carrera de Contabilidad y Auditoría, Chimborazo. mariana.puente@unach.edu.ec. . Orcid ID: 0000-0001-9245-551X

3 Universidad Nacional de Chimborazo, Carrera de Contabilidad y Auditoría, Chimborazo. monica.vallejo@unach.edu.ec. Orcid ID: 0000-0002-4329-5916
} 
positive impact of financial innovation in the generation of income of the financial entities analyzed.

Keywords: income, financial innovation, financial inclusion, financial system

\section{Resumen.}

Introducción: La innovación financiera al ser considerada una herramienta que permite ofrecer servicios y productos financieros más rápidos y a menor costo, se vincula directamente con la generación de ingresos para las entidades del sector financiero. Objetivo: Analizar la incidencia de la innovación financiera en la generación de ingresos en las cooperativas de ahorro y crédito segmento 1 de la provincia de Chimborazo, considerando variables como cajeros automáticos, captaciones, colocaciones, número de operaciones financieras y los ingresos generados en el período 2018 - 2020. Metodología: El enfoque de la investigación fue cuantitativo, el método deductivo inductivo permitió analizar los datos obtenidos de las variables, se aplicó un modelo de regresión lineal a través del software STATA 16.1. Resultados: La innovación financiera denominada cajeros automáticos evidencia un incremento del $36 \%$ de los ingresos de las cooperativas de ahorro y crédito del segmento 1. Conclusión: Según los resultados obtenidos se concluye que existe una incidencia positiva de la innovación financiera en la generación de ingresos de las entidades financieras analizadas.

Palabras claves: ingresos, innovación financiera, inclusión financiera, sistema financiero

\section{Introducción.}

El régimen bancario también conocido como sistema financiero constituye un compendio de acervos, mercados y organizaciones cuya finalidad es la intermediación financiera como un elemento para garantizar la seguridad de inversión de fondos, ahorros, generación de renta y riqueza (Córdoba, 2016), en este contexto el sistema financiero es la base de toda economía, por cuanto a través de este se canalizan recursos para ejecutar operaciones comerciales.

Un sector financiero está caracterizado por instrumentos financieros diversificables, entidades eficientes, una amplia gama de servicios financieros que se integran con la actividad económica, este sector busca la adaptación, evolución de servicios financieros innovadores y un acceso fácil a dichos servicios de la población menos favorecida, en este sentido el papel que juega la inclusión financiera y la innovación financiera se traduce en eficiencia financiera (Chipeta \& Muthinja, 2018).

A nivel global este sistema está conformado por las instituciones, activos financieros cuya misión es garantizar una gestión eficiente de recursos financieros y estabilidad monetaria (Pampillon, et al, 2017). En el caso de Ecuador el sistema financiero en la actualidad lo 
constituyen la banca pública y privada regulada por la superintendencia de bancos y el sector financiero enmarcado dentro de la economías popular y solidaria, cuyo ente de control la Superintendencia de economía popular y solidaria, como manifiesta García (2016) los bancos representan el $90 \%$ de las operaciones de intermediación financiera ecuatoriana.

Al 2020 Ecuador contaba con ochocientas ochenta y siete Cooperativas de ahorro y crédito, clasificadas en cinco segmentos y veinte y cuatro bancos privados, que realizan actividades de intermediación financiera.

Acorde al Código Orgánico Monetario (2019) el sector financiero de la economía popular y solidaria define a aquellas cooperativas de ahorro y crédito cuyos activos sean superiores a 80`000.000 millones de dólares de los Estados Unidos Americanos como segmento 1, segmento 2 aquellas entidades financieras que posean activos entre 20`000.000 USD hasta 80`000.000 USD, segmento 3 activos en un rango de 5 000.000 USD a 20`000.000 USD, segmento 4 rango de activos entre 1`000.0000 USD hasta 5’000.000 USD y el segmento 5 lo conforman aquellas entidades financieras cuyos activos lleguen hasta $1 ` 000.000$ USD.

La Actividad financiera es una prestación o servicio de orden público, controlada y regulada por el Estado y es la representación del sistema financiero nacional, siempre y cuando se cuente con autorización de los entes de control en el marco normativo expedido por la junta de política y regulación monetaria financiera.

En la provincia de Chimborazo a 2020, de acuerdo a los datos obtenidos en la Superintendencia de Economía Popular y Solidaria se muestra que existen cooperativas de ahorro y crédito del segmento 1.

\section{Inclusión Financiera}

En América latina, las instituciones financieras han tendido a diversificar su estructura y con miras a propender profundidad y sostenibilidad financiera, a finales del siglo XX se implementaron reformas financieras enfocadas en controles técnicos, una menor interferencia política, una adecuada administración de riesgos y monitoreo de los procesos de demostración de transparencia en las transacciones bancarias (Vega, et al. 2015)

A nivel mundial la inclusión financiera ha tomado importancia, porque se evidencia la utilidad de esta herramienta en el crecimiento económico, así como mejoramiento de la calidad de vida de los habitantes de un país o región (Anaya \& Romero, 2018), en este contexto el uso de servicios financieros se ha transformado en el aspecto más significativo para los gobernantes porque permite generar una economía igualitaria entre personas y provocando un mayor desarrollo; entre los principales productos y servicios financieros se destacan créditos, cuentas de ahorro, seguros que contribuyen a la generación de inclusión financiera 
En la actualidad la inclusión financiera se ha transformado en un elemento importante de desarrollo porque facilita que los habitantes de una zona geográfica puedan insertarse al sistema financiero formal, incluso superando barreras de espacio y tiempo a través del uso de innovaciones financieras, invertir en salud, negocios financieros y buscar reducir los niveles de pobreza, además permite a los individuos generar una cultura financiera para sobrellevar épocas de crisis como el hecho de perder el empleo, circunstancias imprevistas (Arregui, et al. 2020)

La inclusión financiera juega un papel trascendental en la economía de los países frente a la eliminación de la pobreza, es un paso importante hacia la inclusión de la población más vulnerable a los servicios bancarios en condiciones accesibles (Iqbal \& Sami, 2017)

Autores como Calderón \& Liu (2003), Babajide, et al. (2015) enfocan al concepto de inclusión financiera como la reducción de costos, la disponibilidad formal de créditos y acceso a una cuenta bancaria y estabilidad financiera.

Estudios recientes evidencian que la inclusión financiera es el foco de atención de investigadores, políticos, instituciones financieras, porque su rol se vincula con el fomento del sector financiero, acorde al Banco Mundial para 2020 la inclusión financiera es un elemento primordial para combatir la pobreza, generando un crecimiento económico inclusivo, debido a que la inclusión financiera permite el aceleramiento del crecimiento económico por medio de la asignación de recursos de manera eficiente, reducción de costos de financiamiento, información para aprobación de créditos y gestión de fondos.

El banco mundial define a la inclusión financiera como factor de desarrollo sostenible, que se transforma en una maniobra de inclusión social, en un ambiente favorable, animando competencia, innovación y oferta de servicios financieros seguros.

Con el desarrollo de innovaciones financieras a través del uso de tecnología se genera un espacio para acceder a servicios financieros a un menor costo y riesgo (Banco Mundial, 2020). Con lo antes expuesto se puede concluir que el acceso a servicios financieros ayuda a las familiar y empresas a planificar objetivos a largo plazo, así como emergencias imprevistas como las que en la actualidad se vive por la pandemia COVID-19.

En Ecuador la concepción de inclusión financiera se liga con el propósito de proveer a la población que no cuenta con acceso al mercado financiero la posibilidad de adherirse a los servicios financieros tales como ahorros, créditos, pagos, de acuerdo al Código Monetario y Financiero (2019) del Ecuador en su art. 103 menciona que el Banco Central del Ecuador es el encargado de promover y fomentar la inclusión financiera mediante ampliación del acceso y utilización de servicios financieros formales de la población excluida con limitado acceso a ellos.

El global Index (2017) revela que a nivel mundial el porcentaje de inclusión financiera ha alcanzado el 51\%, lo cual refleja la evolución de dicha inclusión por las innovaciones financieras presentes en el sistema financiero alrededor del mundo que ha permitido la 
digitalización de productos y servicios financieros, en conclusión se muestra que los avances tecnológicos contribuyen al incremento del nivel de inclusión financiera, sin embargo de acuerdo datos estadísticos el nivel de inclusión financiera en Ecuador es del $24 \%$, muy por debajo del índice regional y mundial.

El Global Index, considera varios factores para la inclusión financiera, uno de ellos es el acceso de las personas mayores de 15 años a una cuenta de ahorros, en la tabla 1 se muestra los datos de los países de América del Sur.

Tabla 1

Nivel de acceso a cuentas de ahorro

\begin{tabular}{|c|c|c|c|c|}
\hline País & $\begin{array}{c}\text { Año } 2011 \\
(\%)\end{array}$ & $\begin{array}{c}\text { Año } 2014 \\
(\%)\end{array}$ & $\begin{array}{c}\text { Año } 2017 \\
(\%)\end{array}$ & $\begin{array}{c}\text { Promedio } \\
(\%)\end{array}$ \\
\hline Ecuador & 37 & 46 & 51 & 45 \\
\hline Colombia & 30 & 39 & 46 & 38 \\
\hline Argentina & 33 & 50 & 49 & 44 \\
\hline Chile & 42 & 63 & 74 & 60 \\
\hline Bolivia & 28 & 42 & 54 & 41 \\
\hline Brasil & 56 & 68 & 70 & 65 \\
\hline Uruguay & 24 & 46 & 64 & 44 \\
\hline Perú & 20 & 29 & 43 & 31 \\
\hline
\end{tabular}

Nota: Base de Datos Global Index

Como se evidencia Ecuador en función de la región de América del Sur tiene un nivel de acceso a cuentas de ahorro del $45 \%$ en promedio, muy por debajo del promedio como el de la economía de Brasil con el 65\%, o Chile 60\%, de acuerdo a los datos de Global Index 2017 el promedio de América Latina y el Caribe representan el 54\%, Ecuador está 9 puntos por debajo de dicho promedio.

\section{Barreras de acceso al sistema financiero}

Al ser la inclusión financiera un proceso enfocado a suministrar servicios bancarios como cuentas de ahorro, tipos de crédito, a la población menos favorecida, el objetivo de este tipo de inclusiones es garantizar los servicios financieros a toda la sociedad, sin embargo; existen limitantes en la oferta de servicios financieros tanto para hogares como para empresas, la naturaleza de estas barreras se observa desde el punto de vista de la oferta y la demanda (Roa \&Carvallo, 2018).

El acceso a los servicios financieros es la base del desarrollo económico, fomentando el grado de bancarización de los hogares de menores ingresos económicos, por cuanto la gente de ingresos bajos carece de educación y conocimiento preciso para comprender la disponibilidad de servicios financieros para ellos (Puente, Gavilánez \& Arias, 2020). 
Desde la perspectiva de la oferta las principales barreras para acceder a los servicios financieros son:

- Costos de acceso a los servicios financieros

- Dificultad para asumir riesgos en ciertas operaciones financieras, control de tasas de interés

- Lineamientos vinculados con el lavado de dinero

Desde el punto de vista de la demanda las barreras para acceder a servicios financieros se enfocan en:

- Nivel de ingresos bajos.

- Altos precios en los servicios financieros.

- Insuficiente cultura financiera.

- Factores Culturales (Lee, Wang, Ho, 2020)

Para el caso ecuatoriano, se evidencia barreras de acceso al sistema financiero como la falta de conectividad que impide el acceso a los servicios digitales, brecha de género, instrucción, intergeneracional y los ingresos.

En términos generales el acceso al sistema financiero depende mucho de las circunstancias actuales, porque una gran parte de los gobernantes de la región han otorgado transferencias monetarias a la población más vulnerable con el propósito de mitigar las necesidades elementales de ese sector (Arregui, et a., 2020), en el contexto actual de la pandemia COVID-19 la industria bancaria ha desarrollado productos financieros específicos para los sectores más vulnerables que han sido abatidos en la generación de ingresos durante el confinamiento social.

\section{Innovación Financiera}

La innovación financiera es considerada como el acto de generar nuevos instrumentos financieros basados en tecnología, instituciones y mercados, es un proceso de renovación de un producto o servicios, mejora de canales de seguridad para las transacciones bancarias (León \& Enrique, 2006).

Awrey (2013) define a la innovación financiera como algo diferente que disminuye costos, riesgos o forja un servicio financiero mejorado para la satisfacción de la demanda de los participantes del sistema financiero, en este contexto se concluye que la innovación financiera no es nada más que nuevos productos, procesos, productos e innovaciones institucionales para operación de negocios e implementación de tecnología de la información como la implementación de cajeros automáticos, banca móvil, banca en línea, canales oficiales electrónicos, entre otros.

Acorde al Banco Central Europeo la innovación financiera es una metamorfosis organizacional que admite la disminución de costos y riesgos de las entidades financieras, al igual que la mejora de la calidad de servicios que ofertan a los participantes del sistema financiero (European Central Bank, 2019) 
La innovación financiera en términos de riesgo compartido incrementa la liquidez y tiene un efecto de competencia intenso, su uso para desarrollar mercado de crédito aumenta la asignación eficiente del riesgo crediticio. Grydaki \& Bezemer (2013) han revelado que la innovación financiera incrementa el flujo de liquidez y de los créditos en volumen y por ende se incrementan los ingresos de las entidades financieras.

Estudios como Beck et al. (2016) revelan que la innovación financiera tiene un impacto positivo en las operaciones y eficiencia del sistema financiero, por lo cual la innovación financiera contribuye a un mejor desarrollo y productividad de bancos y cooperativas de ahorro y crédito.

La innovación financiera se centra en tres características como la detención del pasado, mejora del servicio al cliente de las entidades financieras y el incremento de ganancias (Arnaboldi \& Rossignoli, 2015)

La adopción de innovaciones financieras por parte de las entidades financiera en países como Kenia, Reino Unido evidencia un alto potencial de mejoramiento de desempeño financiero y por lo tanto un mejor rendimiento.

El presente estudio busca determinar si en el caso de Chimborazo la innovación financiera incrementa el crecimiento de los ingresos de las entidades financieras del segmento 1

\section{Metodologia.}

La metodología utilizada en la presente investigación se enfoca en dos partes que se detallan a continuación:

a. Revisión de literatura: a través del método deductivo - inductivo se utilizó estrategias de búsqueda para construir el estado del arte como se muestra en la tabla 2.

Tabla 2

Estrategias de Búsqueda

\begin{tabular}{|c|c|c|c|}
\hline Interés de búsqueda & Estrategia & Limitado a & Resultado \\
\hline Financial and & Palabras clave & Año (2014-2020) & 10 \\
\hline Inclusion & $\begin{array}{l}\text { Título } \\
\text { Resumen }\end{array}$ & $\begin{array}{l}\text { Área de interés: } \\
\text { economía, negocios } \\
\text { Idioma: Inqlés }\end{array}$ & \\
\hline Inclusión Financiera & $\begin{array}{l}\text { Palabras clave } \\
\text { Título } \\
\text { Resumen }\end{array}$ & $\begin{array}{l}\text { Área de interés: } \\
\text { economía, negocios } \\
\text { Idioma: Español }\end{array}$ & 9 \\
\hline Financial Innovation & $\begin{array}{l}\text { Palabras clave } \\
\text { Título } \\
\text { Resumen }\end{array}$ & $\begin{array}{l}\text { Año (2014-2020) } \\
\text { Área de interés: } \\
\text { economía, negocios } \\
\text { Idioma: Inglés }\end{array}$ & 25 \\
\hline
\end{tabular}

b. Selección y análisis de datos: una vez concluida la revisión de literatura, se establece el alcance investigativo correlacional, porque se busca determinar si existe un nivel de incidencia de la innovación financiera frente a los ingresos 
generados (captaciones y colocaciones) de las cooperativas de ahorro y crédito del segmento 1 en la provincia de Chimborazo, se consideró una muestra de cinco cooperativas, las cuales cumplen con todos los datos requeridos en el modelo econométrico aplicado. El método deductivo permitió evaluar la evolución de ingresos de las entidades financieras en el período 2018-2020.

\section{Resultados.}

De acuerdo a los datos obtenidos en la Superintendencia de Economía Popular y solidaria, el nivel de ingresos de las cooperativas de ahorro y crédito Segmento 1 del período 2018 - 2020 se muestran en la figura 1, donde se evidencia un crecimiento del $23 \%$ para el año 2019 y un crecimiento del 9\% en 2020 en relación a 2019, el crecimiento registrado en los ingresos de las cooperativas de ahorro y crédito para 2020 está influenciado por la crisis sanitaria en el contexto de la pandemia COVID-19.

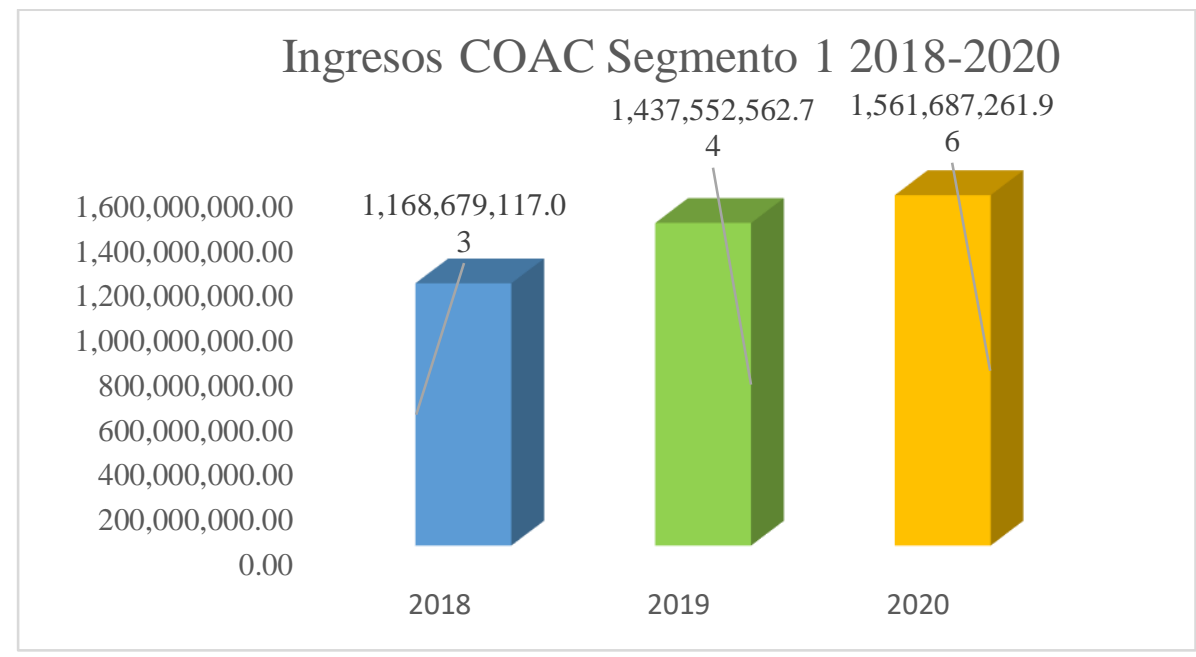

Figura 1 Ingresos COAC's Segmento 1

Nota: Datos obtenidos del Boletín Financiero Mensual SEPS (2020)

\section{Estadísticas descriptivas}

En el análisis descriptivo se utilizó la media, la desviación estándar, mínimo, máximo de cada variable vinculada a la presente investigación, el resumen de los datos se muestra en la tabla 3 .

Tabla 3

Estadísticos Descriptivos

\begin{tabular}{l|rrrrr} 
Variables & Observaciones & Media & Desviación estándar & Mínimo & \multicolumn{2}{c}{ Máximo } \\
\hline ATM & 180 & 2,86 & 3,148352 & 1 & 9 \\
Agencias & 180 & 2,67 & 2,48655811 & 1 & 8 \\
Número de clientes colocaciones & 180 & 437,75 & 448,22 & 6 & 1472 \\
Volumen Colocaciones & 180 & 3172165,81 & 3640328,01 & 22702 & 13879096,9 \\
Número de clientes captaciones & 180 & 47532,65 & 46442,52 & 10264 & 141098 \\
& & & & & \\
\hline
\end{tabular}


Nota: Datos obtenidos de la Superintendencia de Economías Popular y Solidaria y procesados en el programa estadístico STATA 16.1

El promedio de ingresos de las entidades financieras del sector de la economía popular y solidaria del segmento 1 de la provincia de Chimborazo en el período 2018 - 2020 equivalen a 23304496.3 USD (expresado en miles de dólares), en relación al número de clientes de las operaciones de captaciones supera al número de clientes promedio que acceden a un crédito en este tipo de organizaciones financieras, los cajeros automáticos o ATM por sus siglas en inglés tienen una media de 2.86, lo que evidencia que este sector financiero en Chimborazo tiene un nivel de innovación financiera tendiente a reemplazar a las agencias de las cooperativas de ahorro y crédito.

\section{Modelo Econométrico}

Los datos recolectados en un estudio son extensos y no pueden responder a la pregunta de investigación por si solos, por lo tanto, se han procesado y analizado de manera ordenada y coherente. Para la presente investigación se ha utilizado un modelo de regresión lineal como una herramienta que permite relacionar las variables inmersas en el estudio, la ecuación de regresión utilizada para comprobar si la innovación financiera tiene algún grado de incidencia en la generación de ingresos en las cooperativas de ahorro y crédito del segmento 1 en la provincia de Chimborazo.

$$
\text { Ingresos }=\beta_{o}+\beta_{1} X_{1}+\beta_{2} X_{2}+\beta_{3} X_{3}+\beta_{4} X_{4}+\varepsilon
$$

Dónde X1, X2, X3, X4 representan las variables independientes ATM, agencias, número de clientes colocaciones, volumen de créditos, número de clientes captaciones, volumen de captaciones, $\beta$ o es el intercepto o constante; $\beta 1, \beta 2, \beta 3, \beta 4$ representan los coeficientes de las variables independientes, $\varepsilon$ representa el error residual de los valores que no se capturan dentro del modelo econométrico.

\section{Tabla 4}

Modelo Ingresos COAC`s Segmento 1 e innovaciones financieras

\begin{tabular}{|c|c|c|c|c|c|}
\hline Variables & Coeficiente & Error Estándar & $\mathrm{t}$ & $\mathrm{P}$ valor & \\
\hline ATM & 0,36 & 0,1212 & 2,97 & 0,003 & \\
\hline Agencias & 0,13 & 0,1462 & 0,89 & 0,373 & \\
\hline Número de clientes colocaciones & 0,000009653 & 0,00049 & 0,19 & 0,847 & \\
\hline Volumen Colocaciones & $-0,2177$ & 0,1392 & $-1,56$ & 0,12 & \\
\hline Número de clientes captaciones & $-0,3626886$ & 0,3236 & $-1,12$ & 0,264 & \\
\hline Volumen Captaciones & $-0,000027$ & 0,000141 & $-1,97$ & 0,058 & \\
\hline Constante & 25,79 & 5,5156 & 4,68 & 0 & \\
\hline $\mathrm{Fb}(6,168)$ & & & & & 5,18 \\
\hline Probabilidad (F) & & & & & 0,0001 \\
\hline $\mathrm{R}$ cuadrado & & & & & 0,1561 \\
\hline
\end{tabular}


Nota $* \mathrm{p}$ valor 0.05 , si $\mathrm{p}$ valor $>0.05$ no es significativo

De acuerdo a los resultados mostrados en la tabla 4, el 15.61\% de las variaciones de los ingresos generados por las cooperativas de ahorro y crédito del segmento 1 muestran influencia de la variable ATM (considerada una variable de innovación financiera) por cuanto el p valor de dicha variable es menor a 0.05 , convirtiéndose en la única variable significativa.

La analogía entre las variables cajeros automáticos (ATM) e ingresos indica una relación directamente proporcional, porque el coeficiente de regresión muestra que de existir cambio en una unidad de ATM los ingresos de las cooperativas de ahorro y crédito del segmento 1 sufrirían un incremento del $36 \%$, este porcentaje evidencia una incidencia significativa de las innovaciones financieras para generar ingresos en este sector financiero de la economía popular y solidaria segmento 1 de la provincia de Chimborazo.

Al verificar la probabilidad F igual a 0.0001 , la cual es menos al nivel de significancia de 0.05 , por esta razón la hipótesis nula es rechazada y se acepta la hipótesis alternativa, es decir; los ingresos generados por las cooperativas de ahorro y crédito segmento 1 están influenciados por las innovaciones financieras.

\section{Discusión}

Esta investigación revela que el uso de innovaciones financieras en las cooperativas de ahorro y crédito del segmento 1 de la provincia de Chimborazo contribuyen a generar ingresos, este hallazgo se apoya en investigaciones anteriores como Ameme y Wireko (2016); Yusif, (2012) que muestran incidencia de las innovaciones financieras bancarias en los ingresos de la banca, por lo cual estos autores afirman que las entidades financieras deberían buscar el mejor tipo de innovación financiera que contribuya significativamente al desempeño financiero de cada entidad financiera., que estimulen el crecimiento necesario e incremento del rendimiento.

\section{Conclusiones}

- Los ingresos generados por las instituciones financieras del segmento 1 en la provincia de Chimborazo muestran un incremento significativo en los años 2018 - 2020, sin embargo, por el contexto de la pandemia COVID - 19 el crecimiento en 2020 ha sido menor que en años anteriores, en este contexto se evidencio que las innovaciones financieras han contribuido a la generación de ingresos en las cooperativas de ahorro y crédito.

- Los resultados del estudio establecieron una asociación positiva entre la innovación financiera y los ingresos generados por la instituciones financieras, permitiendo concluir que el desarrollo del sector financiero debe fomentar la adopción de nuevos tipos de innovaciones financieras para asegurar la eficiencia financiera y garantizar también el acceso a los servicios financieros de la colectividad, como se muestra en los resultados al analizar el uso de los cajeros automáticos como innovación financiera, se concluye a través del modelo 
econométrico que este tipo de innovación permite mejorar los ingresos del sector financiero segmento 1 de la provincia de Chimborazo en $36 \%$.

\section{Referencias bibliográficas}

Ameme, B. \&Wireko, J. (2016). Impact of technological innovations on customers in banking industry in developing countries. The business and managment review, 7(3), 388-397.

Anaya, A, \& Romero, Y. (2018). La inclusión financiera en Sincelejo (Colombia). Un modelo econométrico probit. Ecos de Economía, 22(46), 91-110.

Arnaboldi, F. \& Rossignoli, B. (2015). Financial Innovation in Banking. Bank Risk, Governance and Regulation, (2004), 127-162.

Arregui, R., Guerrero, R. \% Ponce, K. (2020). Inclusión Financiera y desarrollo. Situación actual, retos y desafíos de la banca. Universidad Espíritu Santo Ecuador.

Awrey, D. (2013). Toward a supply-side theory of financial innovation. Journal Comparative Economics, 41(2), 401-419.

Beck, T., Chen, T., Lin, C., \& Song, F (2016). Financial Innovation: the bring and dark side. Journal of Banking Finance, 72, 28 -51

Calderón, C., \& Liu, L. (2003). The direction of causality between financial development and economic growth. Journal of development economics, 72(1), 321-334.

Muthinja, M. M., \& Chipeta, C. (2018). What drives financial innovations in Kenya's commercial banks? An empirical study on firm and macro-level drivers of branchless banking. Journal of African Business, 19(3), 385-408.

Córdoba, M. (2016). Gestión Financiera. 2ed. Bogotá: Ecoediciones

European Central Bank (2019). Financial innovation for inclusive growth: European approach

Grydaki, M., \& Bezemer, D. (2013). The role of credit in the Great Moderation: A multivariate GARCH approach. Journal of Banking \& Finance, 37(11), 46154626.

Iqbal, B. \& Sami, S. (2017=. Rol of Banks in financial inclusión in Indian. Contaduría y Administración. Vol.62

León, V. \& Enrique, J. (2006). ¿Qué sabe acerca de la innovación financiera? Visión Gerencial (1), 63-71

Pampillo, F., De la Cuesta, M., Ruiz, C., Vásquez, O. \& Bustarviejo, A. (2017). Sistema financiero en perspectiva. Editorial UNED. 
Puente, M, Gavilánez, O. \& Arias, I. (2020). Análisis de la bancarización mediante software como proceso de inclusión financiera en Chimborazo. VII Congreso Internacional SECTEI 2020

Roa, M. \& Carvallo, O. (2018). Inclusión financiera y el costo del uso de instrumentos financieros formales: Experiencia de América Latina y el Caribe. Inter-American Development Bank.

Superintendencia de Economía Popular y Solidaria (2021). Boletines Financieros Mensuales. Obtenido de: https://www.seps.gob.ec/estadistica?boletinesfinancieros-mensuales

Vega, D., Horacio, V \& Reyes, J. (2015). Profundización y sostenibilidad financiera de las bancas de desarrollo rural mexicanas con relación de América Latina. Análisis Económico, XXX(74) 175-193

Yusif, B. (2012). Adopting a specific innovation type versus composition of different innovation types: Case study of Ghanain bank. International Journal of Bank Marketing. 30(3), 218-240. 


\section{PARA CITAR EL ARTÍCULO INDEXADO.}

Puente Riofrío, M. I., Arias González, I. P., \& Vallejo Villalva, M. del P. (2021). Análisis de la innovación financiera como un proceso generador de ingresos en las cooperativas de ahorro y crédito del segmento 1 de la provincia de Chimborazo. ConcienciaDigital, 4(1.2), 187-199. https://doi.org/10.33262/concienciadigital.v4i1.2.1588

\section{$\lfloor$ Ciencia}

El artículo que se publica es de exclusiva responsabilidad de los autores y no necesariamente reflejan el pensamiento de la Revista Conciencia Digital.

El artículo queda en propiedad de la revista y, por tanto, su publicación parcial y/o total en otro medio tiene que ser autorizado por el director de la Revista Conciencia Digital.
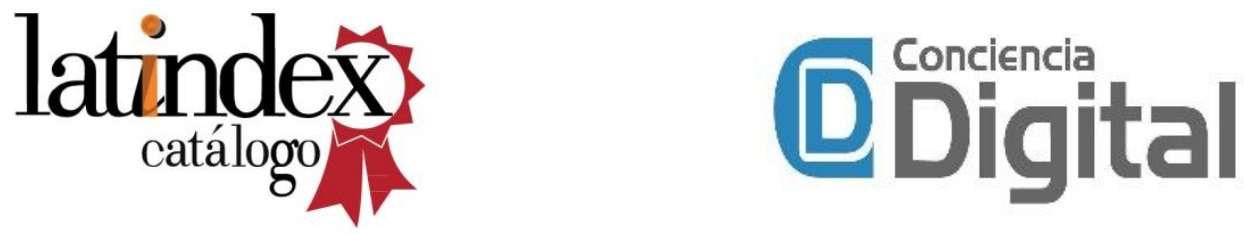